\title{
Predictive Skill for Regional Interannual Steric Sea Level and Mechanisms for Predictability*
}

\author{
IUlita Polkova, ARMin KÖHL, AND DetleF StAMmeR \\ Institute of Oceanography, Center for Earth System Research and Sustainability, University of \\ Hamburg, Hamburg, Germany
}

(Manuscript received 26 November 2014, in final form 29 June 2015)

\begin{abstract}
Based on decadal hindcasts initialized every five years over the period 1960-2000, the predictive skill of annual-mean regional steric sea level and associated mechanisms are investigated. Predictive skill for steric sea level is found over large areas of the World Ocean, notably over the subtropical Atlantic and Pacific Oceans, along the path of the North Atlantic Current, and over the Indian and Southern Oceans. Mechanisms for the predictability of the thermosteric and halosteric contributions to the steric signal are studied by separating these components into signals originating from processes within and beneath the mixed layer. Contributions originating from below the mixed layer are further decomposed into density-related (isopycnal motion term) and density-compensated (spice term) changes. In regions of the subtropical Pacific and Atlantic Oceans, predictive skill results from the interannual variability associated with the contribution from isopycnal motion to thermosteric sea level. Skill related to thermosteric mixed layer processes is found to be important in the subtropical Atlantic, while the spice contribution shows skill over the subpolar North Atlantic. In the subtropics, the high predictive skill can be rationalized in terms of westward-propagating baroclinic Rossby waves for a lead time of $2-5 \mathrm{yr}$, as demonstrated using an initialized Rossby wave model. Because of the low Rossby wave speed in high latitudes, this process is not separable from the persistence there.
\end{abstract}

\section{Introduction}

Global-mean sea level rise is often used as an important indicator of anthropogenic climate change, implying that natural fluctuations represent a significantly smaller contribution to the global sea level changes than the anthropogenic forcing of Earth's energy budget (Huber and Knutti 2011; Santer et al. 2011). On the other hand, regional sea level variability can be very different from the global mean and is strongly influenced by internal climate variability (Delworth and Knutson 2000; Zhang and Church 2012; Stammer et al. 2013). Previous studies indicate that interannual-to-decadal sea surface height ( $\mathrm{SSH}$ ) changes are mostly steric in nature and are controlled by wind (through Rossby

\footnotetext{
* Supplemental information related to this paper is available at the Journals Online website: http://dx.doi.org/10.1175/JCLI-D-14-00811.s1.

Corresponding author address: Iuliia Polkova, Institute of Oceanography, Center for Earth System Research and Sustainability, University of Hamburg, Bundesstr. 53, 20146 Hamburg, Germany.

E-mail: iuliia.polkova@uni-hamburg.de
}

wave and Ekman pumping dynamics) and buoyancy forcing (Church et al. 2010; Piecuch and Ponte 2011; Stammer et al. 2013). However, Vinogradova et al. (2007) showed that, for high latitudes and over coastal areas, the contribution of mass redistribution to regional sea level changes cannot be ignored. Because future regional sea level changes are seen as a major threat to all low-lying coastal areas around the world (Church et al. 2010), it is desirable to establish a firm understanding of the predictability of regional sea level on interannual to decadal time scales and beyond. However, understanding mechanisms of regional sea level variations and their predictability in detail remains a challenging task because in reality all involved forcing factors may act in conjunction, and the response of sea level can be a complex combination of different processes to related local and nonlocal forcing effects.

Interannual-to-decadal climate prediction studies represent a relatively new scientific field, which has a practical goal of providing information on the climate system up to decadal time scales to social and economic institutions, and the more scientific goal of understanding interannual variability and predictability of the climate 
system. In addition to the predictive skill due to external forcing, initializing decadal predictions from the observed state provides predictive skill for various components of the climate system (Smith et al. 2007; Matei et al. 2012; Pohlmann et al. 2013; Boer et al. 2013). Nevertheless, precise mechanisms that could explain the predictive skill up to decadal time scales are yet to be understood. Also, the predictive skill has not been tested for a suite of climate parameters. This holds in particular for sea level, which was addressed only in a few studies. On longer than decadal time scales, the potential for predictability of regional interannual SSH was investigated by Griffies and Bryan (1997), while Polkova et al. (2014) presented the first analysis of predictive skill for regional interannual SSH from initialized decadal hindcasts. Predictive skill for seasonal sea level forecasts was recently analyzed by Miles et al. (2014).

Piecuch and Ponte (2011) advocate that interannual steric SSH variability is mostly wind-driven rather than related to local surface buoyancy fluxes and suggest that the sea level signal therefore might not be predictable at all, as we cannot predict wind stress changes. On the other hand, if sea level anomalies are built up by wind stress anomalies that persist over some time, a substantial fraction of this variability might result from propagating baroclinic Rossby waves and, at their characteristic time scales, should still be predictable. In support of this hypothesis, propagating baroclinic Rossby waves were shown previously to be responsible for interannual-to-decadal variability in the $\mathrm{Pa}$ cific and Atlantic Oceans (Sturges et al. 1998; Fu and Qiu 2002; Cabanes et al. 2006).

In this paper, we continue the work of Polkova et al. (2014) by focusing on the predictive skill of the interannual steric sea level component with the aim of finding mechanisms underlying the decadal predictability. Understanding the processes contributing to sea level variability can provide confidence in the predictive skill for sea level and related climate variables, such as ocean heat content changes and freshwater content changes. Hence, we seek to identify regions and time scales of predictability for thermosteric SSH changes (because of ocean heat content changes) and halosteric SSH changes (because of freshwater content changes) and their contributions. Köhl (2014) suggests that outside of high latitudes and mode water regions, thermosteric SSH variability is dominated by isopycnal heaving that is associated with wind forcing. Here, we analyze the extent to which predictive skill for steric SSH is related to the Rossby wave mechanism. For this, we use a simple baroclinic Rossby wave model based on the knowledge of initial conditions (Qiu and Chen 2006). In addition, because the thermosteric isopycnal motion term is related to Rossby waves, this mechanism might explain predictive skill for the thermosteric SSH changes. For the halosteric SSH changes, the component related to the advection of spiciness by the mean currents dominates the signal and might provide long-term predictability (Köhl 2014).

The structure of the remaining paper is organized as follows: Section 2 briefly describes the coupled oceanatmosphere model and experiments performed to study interannual steric SSH predictability. This section also describes the verification metrics and datasets. Section 3 contains an overview of predictive skill for sea level changes based on the tide gauges dataset, and section 4 describes the skill for steric sea level and its thermosteric and halosteric contributions based on the ocean synthesis. Section 5 introduces a Rossby wave model as a mechanism for predictive skill of sea level, reviews the skill related to the Rossby wave mechanism, and discusses the relative contribution from isopycnal heaving and spiciness to sea level predictability. Finally, conclusions and a discussion are presented in section 6 .

\section{Methodology}

\section{a. The coupled model}

The ocean model used in this study is the University of California, Los Angeles, coupled general circulation model (UCLA CGCM), which consists of the UCLA atmospheric general circulation model (UCLA AGCM, version 7.1, with a horizontal resolution $2.5^{\circ} \times 2^{\circ}$ and 29 vertical layers; Mechoso et al. 2001) coupled with the Massachusetts Institute of Technology ocean general circulation model (MITgcm, with the horizontal resolution $1^{\circ} \times 1^{\circ}$ and refinement of the meridional resolution toward the equator and 46 vertical layers; Marshall et al. 1997). The experimental setup is described in detail by Polkova et al. (2014). The coupled model was used previously by Cazes-Boezio et al. (2008) for simulation of El Niño-Southern Oscillation and by Stammer et al. (2011) and Agarwal et al. (2014) to investigate the coupled sea level response to Greenland ice melting. Ma et al. (2013) used the model to study the connection between land surface processes and the tropical climate.

\section{b. Experiments}

The initialized hindcasts were performed according to the protocol from phase 5 of CMIP (CMIP5; Taylor et al. 2012). Hence, ensembles of initialized hindcasts (retrospective predictions) are initialized every five years from the GECCO ocean synthesis at the end of December 1960, $1965,1970, \ldots, 2000$, with three realizations for each starting date. Three ensemble members are generated using 2-day atmospheric lagged initialization. The GECCO synthesis ( $1^{\circ}$ horizontal resolution and 23 vertical layers) is 
available from the German contribution to Estimating the Circulation and Climate of the Ocean (GECCO) project (Köhl and Stammer 2008a,b) and covers the period 1952-2001. The GECCO synthesis uses the adjoint method to bring the model into consistency with the NCEP atmospheric state (Kalnay et al. 1996), satellite observations, and the temperature and salinity profiles from the World Ocean Database.

As ocean initial conditions for the initialized hindcasts, we used 3D temperature, salinity, zonal and meridional velocities, and SSH fields. For atmospheric initial conditions, a 50-yr-long assimilation run was carried out, in which the ocean component of the coupled model was reinitialized monthly from the ocean GECCO state over the period 1952-2001. Note that although the MITgcm is used for the GECCO production and is also a part of UCLA CGCM used for the initialized hindcasts, the configurations of the ocean model are different. GECCO and the initialized hindcasts also use different forcings. GECCO estimated the forcing to minimize the differences to ocean data. Therefore, the biases of GECCO are much smaller than those of the coupled model. Introducing GECCO initial conditions into the coupled model will still lead to initialization shocks despite the fact that both models are based on the same MITgcm.

\section{c. Initialization method}

By initializing predictions close to the state of the real world, a dynamical model attempts to trace out the actual future evolution of the climate system based on the information contained in the initial conditions. During the model integration, the state of the model will diverge from the initial state, giving rise to model bias (Stockdale 1997; Pierce et al. 2004). To deal with model drift, decadal prediction studies have tested several initialization schemes such as anomaly initialization, full-field initialization and full-field initialization with momentum and/or buoyancy flux correction (e.g., Smith et al. 2007, 2013; Magnusson et al. 2013). To date, there is no common agreement on what is the best practice for initializing decadal predictions. In our experimental setup (Polkova et al. 2014), the full-field initialization employing heat and freshwater flux correction (FC-HIND) showed the best result for predictive skill of annual-mean sea surface temperature (SST) and sea level, followed by full-field initialization and anomaly initialization. The SST skill over the North Atlantic and the extratropical Southern Hemisphere was related to the ability of FC-HIND to better represent the mixed layer depth (MLD) than the other two initialization methods.

Hence, FC-HIND are performed with monthly climatological-mean corrections to the surface heat and freshwater fluxes. For this, a reference 10-yr run over 1997-2006 was carried out in which the SST and sea surface salinity of the coupled model were relaxed toward the monthly climatology of GECCO. The patterns of correction terms did not show sensitivity to a longer nudging run and different time slice. The correction terms were calculated as a difference in the associated flux climatology between the reference run and GECCO. The flux correction reduces the drift in the analyzed variables but not completely; thus, an a posteriori bias correction [as described by Smith et al. (2013)] is applied to hindcasts.

\section{d. Verification metrics}

To estimate how fast initialized hindcasts forget about their initial state, we performed the predictive skill estimation based on the GECCO dataset. To this end the anomaly correlation coefficient $\left(\mathrm{COR}_{t}\right)$ is used to measure predictive skill for decadal hindcasts as a function of lead time. It is defined as

$$
\mathrm{COR}_{t}=\frac{\sum_{s} h_{s t} o_{s t}}{\sqrt{\sum_{s} h_{s t}^{2} \sum_{s} o_{s t}^{2}}}
$$

where $h_{s t}$ is the ensemble mean of predicted anomalies at lead time $t$ and starting date $s$, and $o_{s t}$ are the corresponding GECCO anomalies. In all cases, anomalies are calculated with respect to the mean over 1952-2001; for the predicted anomalies, the mean is taken from the assimilation run to provide the same reference for all hindcasts. We base our investigations on annual SSH anomalies and analyze the skill for the first lead year (yr1) and the averages over four lead years 2-5 and 6-9 (yr2-5 and yr6-9) as in the verification framework for the CMIP5 experiments described by Goddard et al. (2013). To focus on the skill due to initialization, the hindcasts and GECCO fields were detrended prior to computing correlation coefficients (Polkova et al. 2014).

To demonstrate that initialized predictions show enhanced predictive skill beyond persistence, a correlation skill for initialized hindcasts is compared with the lowskill persistence forecast. For persistence skill shown in yr1, SSH anomaly at a year that precedes initialization is extended into the future. For persistence skill shown for averaged periods (yr2-5 and yr6-9), an average of SSH anomalies over four years (i.e., lag5 and lag9) that precede initialization is used. An estimation of the significance level for $\mathrm{COR}_{t}$ is based on the nearest-neighbor bootstrap method (Goddard et al. 2013).

\section{e. Verification datasets}

To estimate actual predictive skill of decadal hindcasts, the verification metrics should be based on observations 


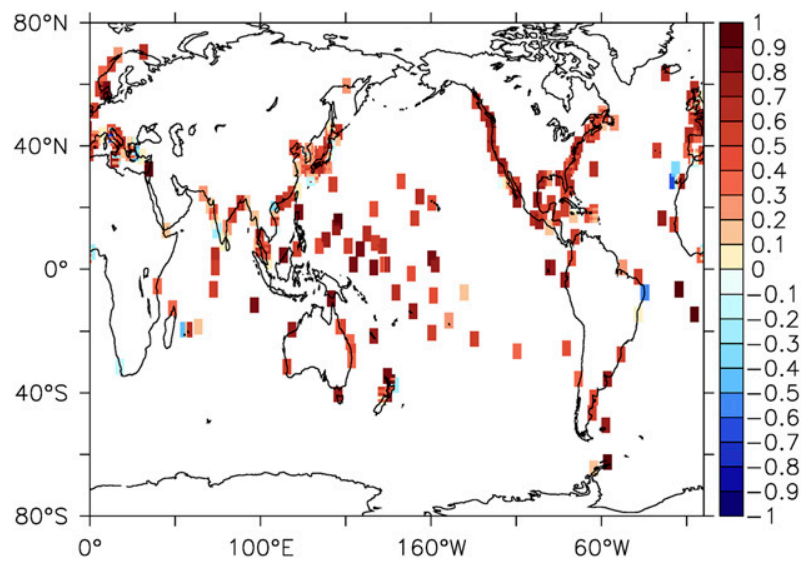

FIG. 1. Correlation between the detrended annual-mean GECCO SSH changes and the tide gauge data, which were assimilated in GECCO. Correlation coefficients are calculated over the period 1952-97. Tide gauge data are corrected for the inverted barometer response (based on the NCEP reanalysis pressure).

(Goddard et al. 2013; Boer et al. 2013). However, such verification is difficult to implement for some important climate parameters like sea level or the Atlantic meridional overturning circulation because of the relatively short or sparse observational records. For this study, we used the tide gauge dataset, the coverage of which is restricted to continental boundaries and oceanic islands (Ponte 2006).

In the studies by Pohlmann et al. (2009, 2013), Kröger et al. (2012), and Kim et al. (2012), the verification of decadal predictions was performed with respect to reanalysis/synthesis datasets. We also base our analysis of the predictive skill for steric SSH changes and its contributions on the GECCO synthesis such that current predictive skill evaluation does not account for the errors in ocean initial conditions. To account for errors in ocean initial state, ensemble predictions based on oceanic perturbations are needed (e.g., Hawkins et al. 2011).

A comparison of the annual-mean sea level of GECCO with tide gauge data in terms of correlation coefficients is shown in Fig. 1. High correlation values (COR $>0.5)$ are shown over the western and eastern coasts of the United States, Europe, and near the islands in the tropical Pacific Ocean. The comparison of GECCO with altimeter observations was performed by Köhl and Stammer (2008a), who showed that the amplitude and pattern of the SSH trend closely match the changes observed in the AVISO dataset during 1992-2001. In terms of interannual sea level variability, the patterns of GECCO variability are comparable to those of the AVISO variability, except for the high latitudes, where GECCO underestimates the variability, and western boundary currents, where GECCO overestimates the variability. The figures of sea level variability and its thermosteric and halosteric contributions are provided in the supplemental material and are very similar to those shown by Köhl (2014), who investigated mechanisms leading to interannual regional SSH variability based on the second version of the GECCO ocean synthesis (GECCO2).

\section{Predictive skill for SSH changes based on tide gauges data}

As was mentioned in section 2e, the GECCO synthesis is in general in good agreement with the tide gauge data, which are assimilated in the synthesis. To see if the initialized hindcasts reproduce the observed sea level variability, the SSH predictive skill estimated with respect to the tide gauge dataset for yr1, yr2-5, and yr6-9 is shown in Fig. 2. In the first lead year, the SSH hindcasts are in agreement with tide gauge data over the eastern coast of the United States and Eurasian continent and in the tropical Pacific Ocean. Less agreement is evident over the coasts of India and northern Europe and the western coast of the United States. For yr2-5, the decrease of correlation is evident along the continental coasts, while some high correlation values remain near $20^{\circ} \mathrm{S}$ and $20^{\circ} \mathrm{N}$ in the Pacific Ocean and in the Caribbean basin.

\section{Predictive skill for steric SSH changes based on the ocean synthesis}

Regional steric $\mathrm{SSH}$ is derived by integrating density anomalies over a column of seawater. Calculating density anomalies with respect to the time-mean salinity and temperature fields allows for the estimation of sea level anomalies due to temperature changes (thermosteric SSH) and salinity changes (halosteric SSH), respectively (Pattullo et al. 1955).

Since most low-frequency SSH variability is associated with steric SSH changes, we focus in the following on the predictive skill for the steric SSH changes. Respective patterns of skill are shown in Fig. 3 for yr1, yr2-5, and yr6-9. For lead year one, the correlation values are high all over the ocean due to the closeness of hindcasts to their initial conditions, which is also seen in the persistence skill. For yr2-5 and yr6-9, representing the interannual time scale, patterns of high skill are evident in the Indian Ocean, Southern Ocean, and the subtropical Atlantic and Pacific Oceans. These patterns of skill are very similar to those for dynamic topography shown by Polkova et al. (2014). Because the correlation coefficient is insensitive to the amplitude of analyzed anomalies, we additionally analyzed the root-mean-square error skill score, which represents the relative accuracy of the initialized hindcasts with respect to uninitialized ones 


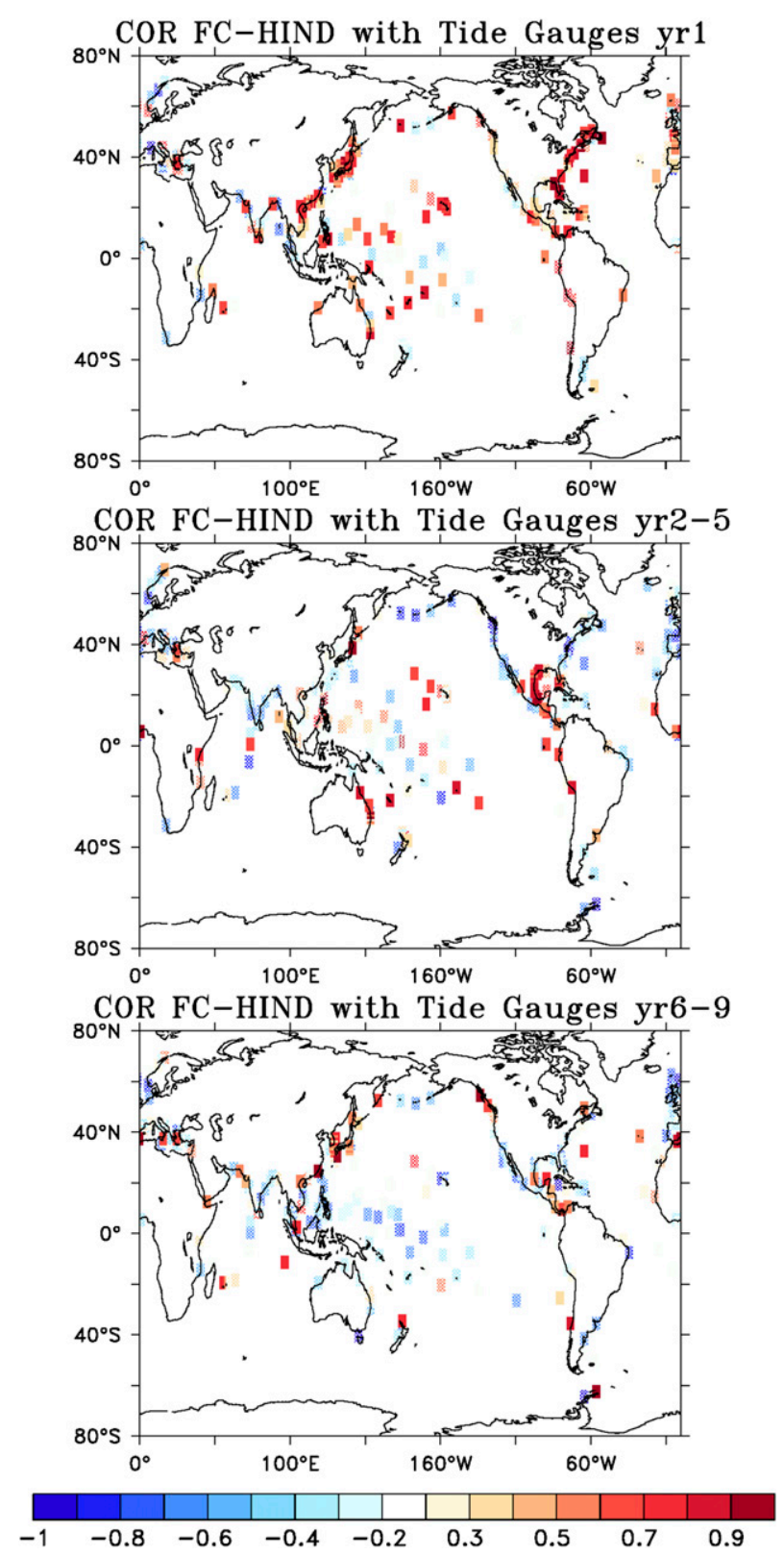

FIG. 2. COR skill calculated between the detrended tide gauges data and initialized SSH hindcasts at (top) yr1, (middle) yr2-5, and (bottom) yr6-9. Insignificant COR values are white dotted. The $90 \%$ significance level is estimated with the bootstrap method.

(Goddard et al. 2013). The patterns of the root-meansquare error skill score for steric SSH anomalies, where initialized hindcasts demonstrate smaller errors, are in agreement with the areas of high correlation skill (Fig. S3 in the supplemental material).

Persistence skill is significant over the Indian Ocean and Southern Ocean up to yr2-5. In general, persistence shows lower values of skill in the range of about $0.4-0.5$. While different from the hindcasts' skill, persistence skill in the equatorial Atlantic is high for yr1, and in the equatorial Pacific persistence skill remains significant until yr2-5.

To demonstrate how much predictability the thermosteric and halosteric contributions possess individually, the COR skill is plotted separately for thermosteric and halosteric SSH changes in Fig. 4. It reveals that both thermosteric and halosteric terms have large-scale patterns of high predictive skill up to yr6-9. Both terms show the skill in the extratropical Southern Hemisphere and quite similar skill patterns in the Atlantic Ocean for yr6-9. In addition, the thermosteric signals have skill in the Indian Ocean and the subtropical Pacific. In the tropical North Atlantic and in some areas of the eastern North Pacific for yr2-5, both components show skill that is not present in the total steric signal. This might be related to predictable elements of density-compensated temperature-salinity anomalies (spice term) that have no effect on steric SSH changes. Though the skill at yr2-5 and yr6-9 for the halosteric term is high, the contribution of halosteric SSH variability to the steric signal is much smaller than that of thermosteric SSH. The regions of high variability for thermosteric and halosteric components are consistent with those previously reported by Köhl (2014) and provided in the supplemental material. The subpolar North Pacific and subpolar North Atlantic are the regions where the halosteric term shows predictive skill and where the GECCO synthesis shows high interannual variability (about $20 \mathrm{~cm}$ ).

The skill for the thermosteric term reflects predictability of ocean heat content (OHC) changes. However, the relation between thermosteric SSH and $\mathrm{OHC}$ changes can be complicated by the nonlinear dependence of density on temperature (Ivchenko et al. 2008). Matei et al. (2012) analyzed the predictive skill for OHC using an initialized decadal prediction approach. They advocate that over the tropical and extratropical North Atlantic and over the central North Pacific for yr2-5, the persistence of $\mathrm{OHC}$ anomalies, which are induced by climate modes such as Atlantic multidecadal oscillation and Pacific decadal oscillation, is responsible for predictive skill. Yeager et al. (2012) related the skill for OHC changes over the subpolar North Atlantic gyre to the correct representation of large-scale heat advection attributed to ocean circulation anomalies. In the following section, we will investigate in more detail the predictive skill associated with OHC changes and freshwater content changes and the contribution of propagation signals to the predictive skill considering a very simple Rossby wave model.

\section{Predictability mechanisms}

\section{a. Predictability due to the Rossby wave mechanism}

Previous results showed that the first baroclinic mode waves take, depending on latitude, from several months to 


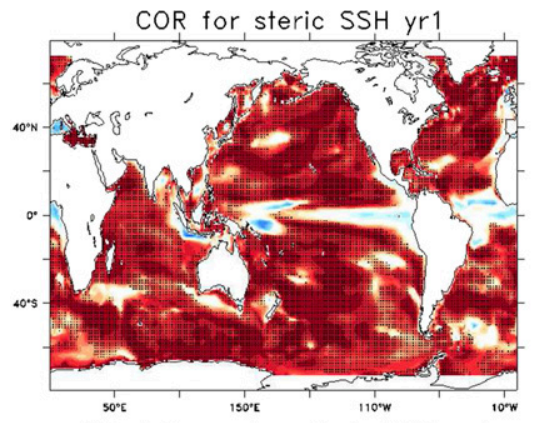

Persistence for steric SSH yr

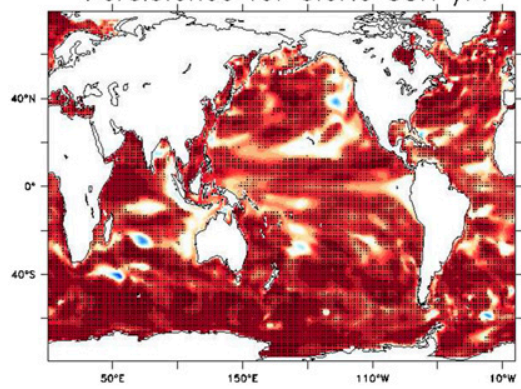

Cross-COR for steric SSH (yr1)

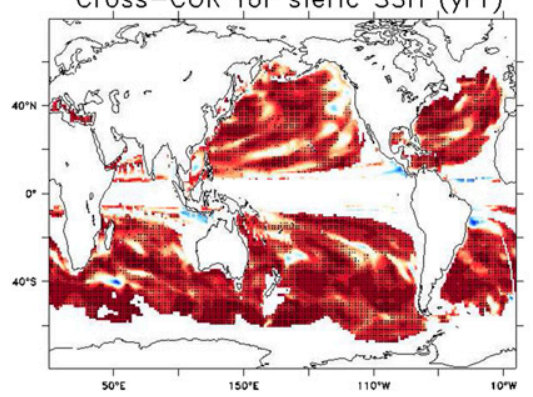

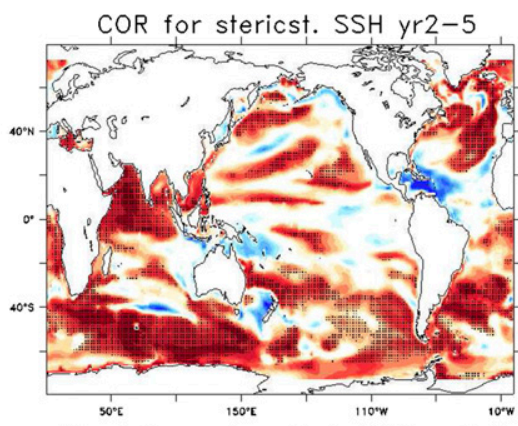

Persistence for steric SSH yr2-5

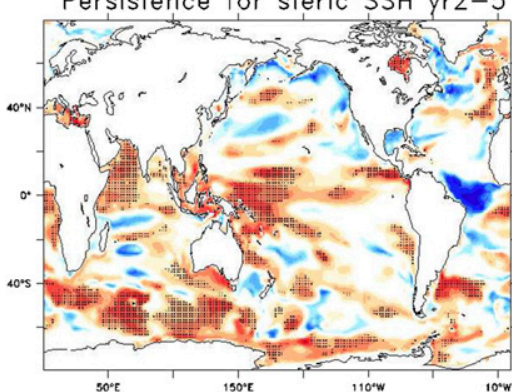

Cross-COR for steric SSH (yr2-5)

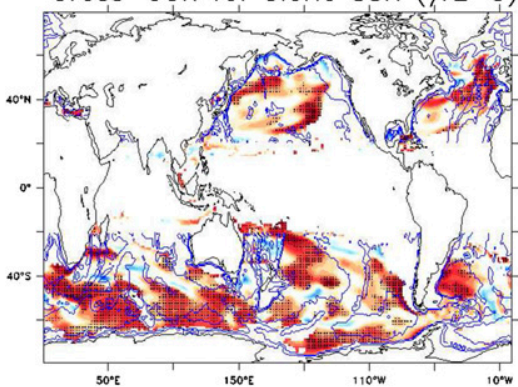

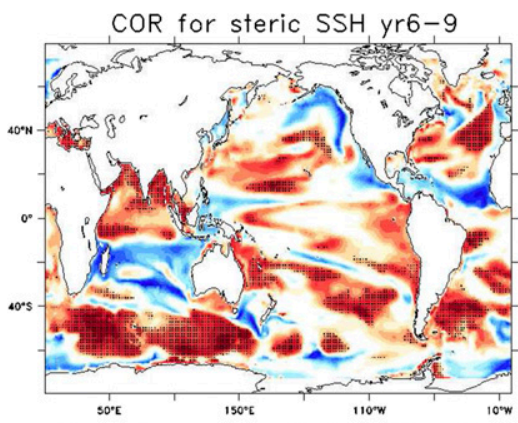

Persistence for steric SSH yr6-9

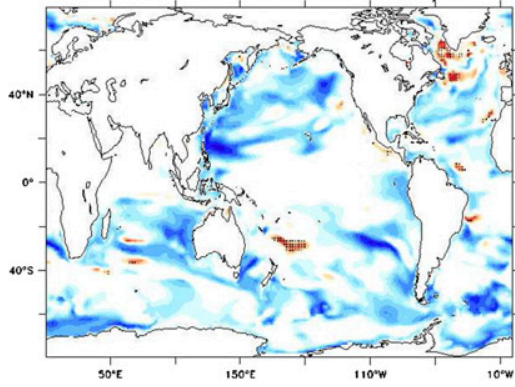

oss-COR for steric SSH (yr6-9)

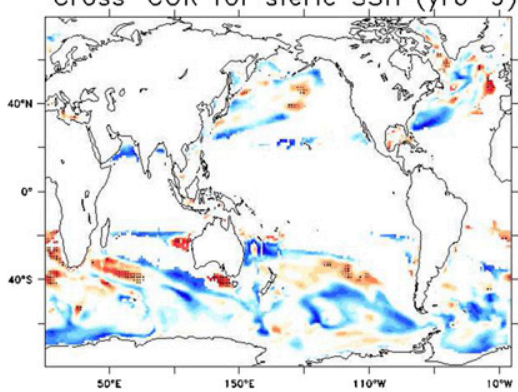

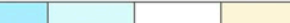

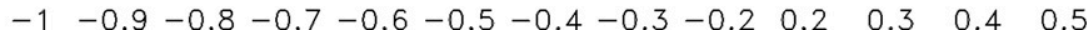

0.6

0.7

$0.8 \quad 0.9$

FIG. 3. (top) COR skill calculated between detrended GECCO and initialized hindcasts and (middle) the persistence skill derived from GECCO for steric SSH changes at (left) yr1, (center) yr2-5, and (right) yr6-9. Hatched grid boxes show COR values that are significant at the $90 \%$ level, estimated with the bootstrap method. (bottom) Predictive skill due to the Rossby wave mechanism $\left(\mathrm{CCOR}_{t}\right)$ at yr1, yr2-5, and yr6-9. Calculation of $\mathrm{CCOR}_{t}$ is based on the detrended steric SSH changes derived from GECCO. Topography is overlaid on the cross-correlation pattern for yr2-5 as contours with the interval of $1500 \mathrm{~m}$.

decades to cross an ocean basin (e.g., Chelton et al. 1998; Yang et al. 2003; Qiu and Chen 2006). The difference in cross-basin time for a particular mode of Rossby wave is a result of the latitudinal variation of the phase speed. Latif and Barnett (1996) advocate that, in the North Pacific, the variability of thermal anomalies is attributed to Rossby wave adjustment and to the advection of water from the subtropical mixed layer around the subtropical gyre. Weijer et al. (2013) proposed that decadal variability in the subpolar North Pacific might result from the pressure (density) adjustment to Rossby wave fronts arriving at the western boundary. Capotondi and Alexander (2001) found that, for a latitudinal band $10^{\circ}-15^{\circ} \mathrm{N}$, long baroclinic Rossby waves, in the $7-10-\mathrm{yr}$ period band and with the propagation speed of about $13 \mathrm{~cm} \mathrm{~s}^{-1}$, might be responsible for decadal variability.

Motivated by these studies, we analyze predictive skill for steric SSH predicted by the Rossby wave mechanism. To see whether FC-HIND represents westward-propagating SSH anomalies comparable to those of GECCO, the Hovmöller diagram for annual SSH anomalies at $40^{\circ} \mathrm{S}$ in the Pacific Ocean is shown in Fig. 5. In the diagram for FC-HIND, the first five years of predicted SSH anomalies are merged together, that is, 1961-65, 1966-70, . ., 199095. The westward-propagating SSH anomalies in FCHIND show correspondence to those from GECCO in the first lead years (e.g., negative SSH anomaly over 1971-75 and 1976-80), indicating that wave propagation 

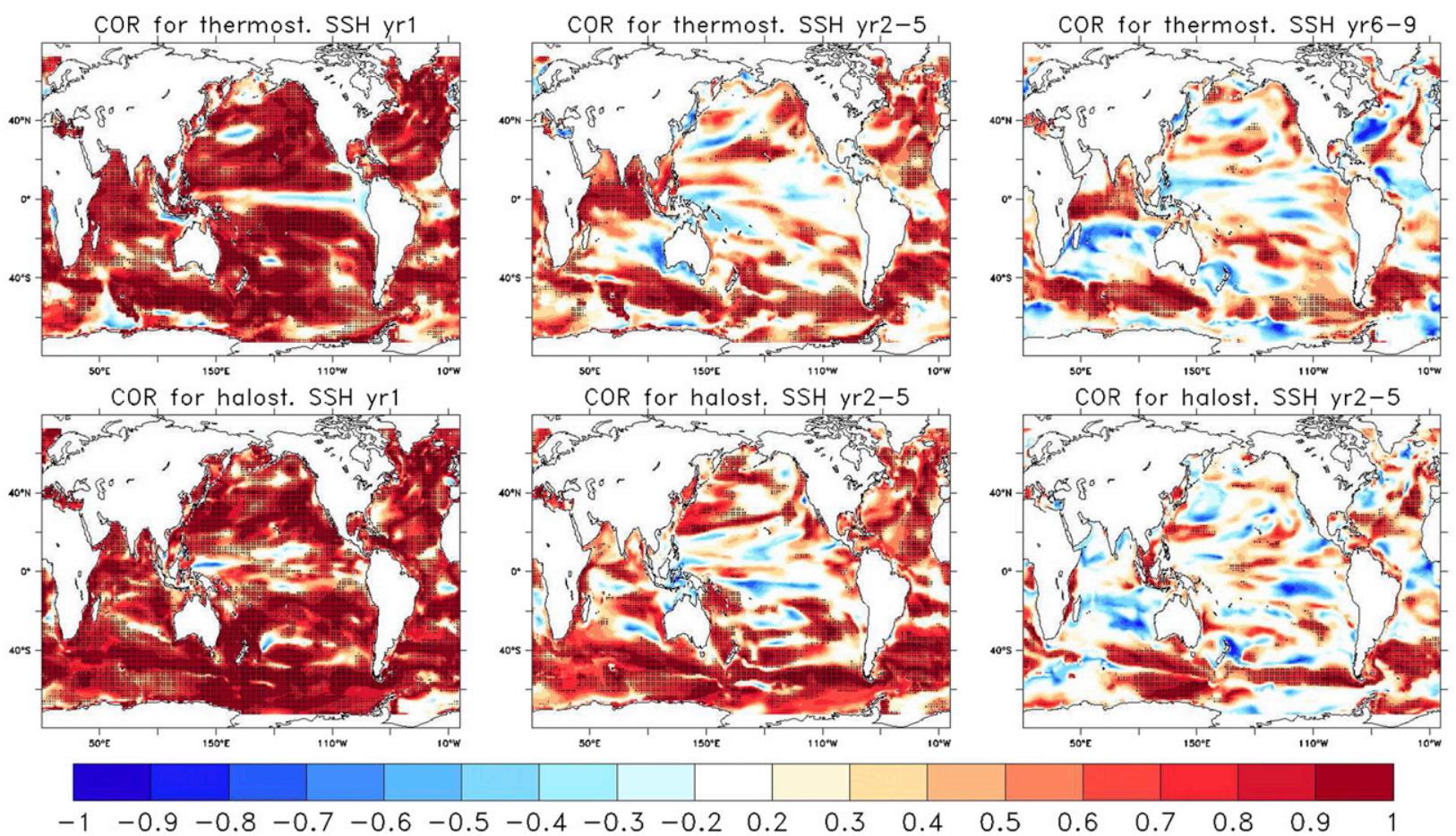

FIG. 4. COR skill for detrended (top) thermosteric and (bottom) halosteric SSH changes at (left) yr1, (center) yr2-5, and (right) yr6-9. Hatched region shows COR values that are significant at the $90 \%$ level, estimated with the bootstrap method.

might provide a predictable signal. Because propagating SSH anomalies can be further damped or excited along the propagating pathways by wind forcing, which differs in GECCO and FC-HIND, their propagating features can develop differently (e.g., SSH anomaly at $80^{\circ} \mathrm{W}$ initialized in 1965 and at $120^{\circ} \mathrm{W}$ initialized in 1990).

The assumption of the Rossby wave model (RWM) is that the signal related to the initial conditions provided by GECCO is simply propagated westward with the constant Rossby wave speed of the first baroclinic mode. The phase speed of the long baroclinic Rossby wave $\left(c_{R}\right)$ is calculated using the Wentzel-Kramers-Brillouin (WKB) approximation as discussed by Chelton et al. (1998). A figure with the estimated phase speed values is provided in the supplemental material. To evaluate how much skill of the climate model might be related to the propagation of Rossby waves, the pointwise crosscorrelation function is calculated between the steric sea level $\eta_{s t}$ at lead year $t$ and the steric sea level $\eta_{s 0}$ at the year that precedes initialization at the location $x_{0}$, from which a mode-one Rossby wave would bring the signal to the location at lead year $t$ :

$$
\mathrm{CCOR}_{t}=\frac{\sum_{s} \eta_{s t} \eta_{s 0}}{\sqrt{\sum_{s} \eta_{s t}^{2} \sum_{s} \eta_{s 0}^{2}}},
$$

where index $s$ corresponds to start dates. The longitude of $\eta_{0}$ is found as $x_{0}=x_{t}+c_{R} t$ with phase speed $c_{R}$ in degrees per year at location $x_{t}$.

For the consistency with previous figures, the crosscorrelation function in Fig. 3 (bottom panel) is shown for $\mathrm{yr} 1, \mathrm{yr} 2-5$, and yr6-9. We are looking for the regions where the RWM shows larger skill than the persistence (Fig. 3, middle). To this end, in the eastern North Atlantic and subtropical Pacific at yr2-5, the cross-correlation function demonstrates a resemblance to the pattern of predictive skill for steric SSH (Fig. 3, top). The pattern correlation coefficient, which provides a measure for the level of correspondence between predictive skill for steric SSH and skill from RWM, is about 0.44 . At yr6-9, the simple Rossby wave model does not show significant skill except for a very small band near $40^{\circ} \mathrm{S}$ in the eastern half of the Indian and Pacific Ocean. In high latitudes, where Rossby wave speed is only few degrees per year, the persistence model has similar skill to the RWM. In some regions, for instance, north of New Zealand and around $120^{\circ} \mathrm{W}$ in the South Pacific, a sharp transition from high to low correlation is evident. This is related to steep topographic features, which are not considered in the RWM but may block the Rossby waves.

In current analysis, the predictability associated with the RWM is limited to yr2-5. One of the reasons for this could be the assumption used for calculating the phase speed. For instance, the propagation based on the mean 

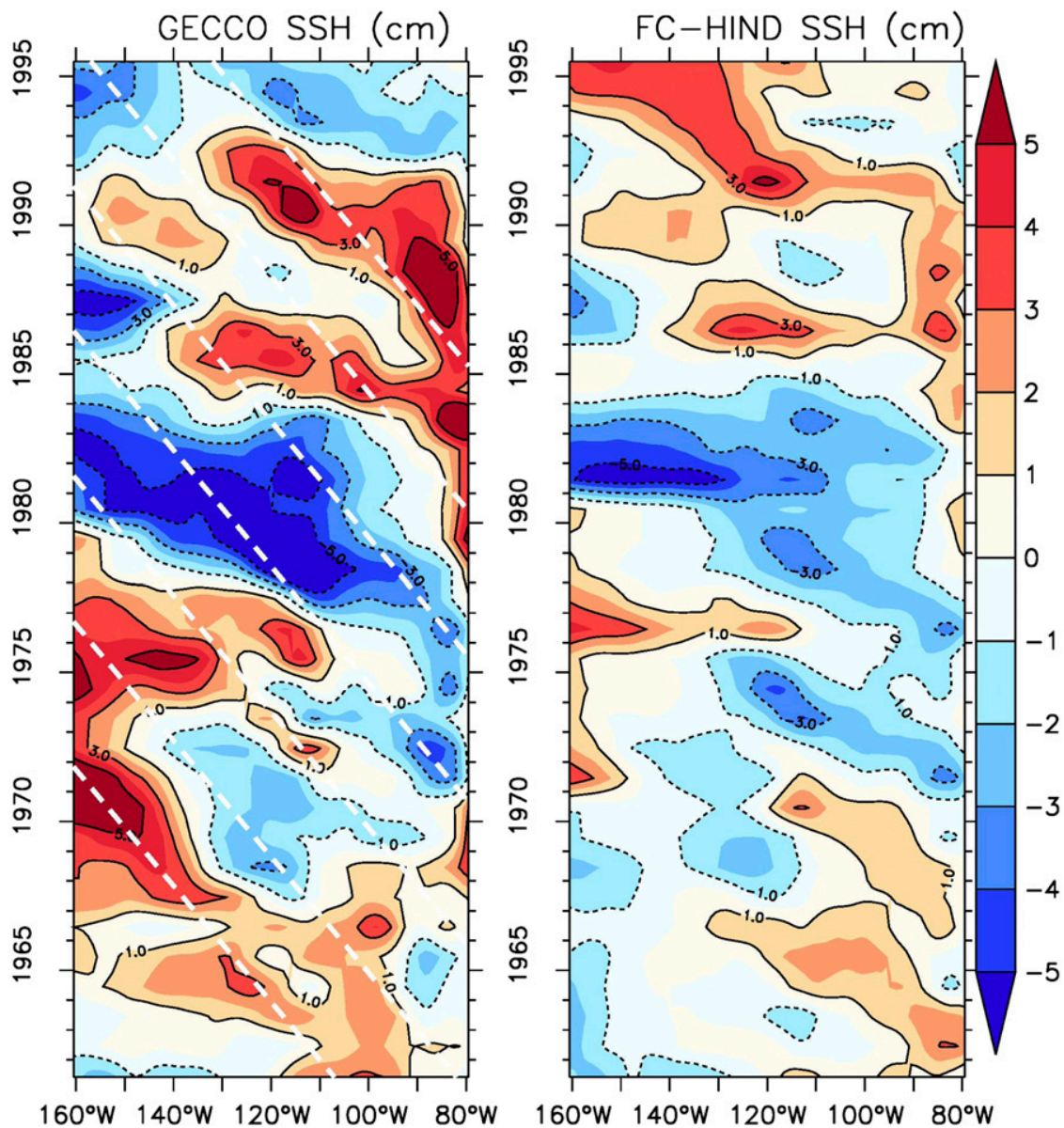

FIG. 5. Time-longitude (Hovmöller) diagram for annual SSH anomalies from (left) GECCO and (right) initialized hindcasts over 1961-95 at latitude $40^{\circ} \mathrm{S}$. For the hindcasts, the first 5-yr periods of the initialized hindcasts were merged together, that is, 1961-65, 1966-70, . ., 199195. Dashed white lines show propagation pathways according to the simple first-mode baroclinic Rossby wave model at latitude $40^{\circ} \mathrm{S}$.

phase speed at $40^{\circ} \mathrm{S}$ in Fig. 5 indicates that the observed SSH anomalies propagate faster than those proposed by the simple RWM, suggesting potential effects of other processes. The fact that planetary waves propagate faster than the linear theory predicts was discussed in many studies. In this respect, Killworth and Blundell (2003a,b) reconsidered the WKB assumption about zero background mean flow and showed that including the slowly varying mean flow and topography resolves the problem of substantial differences in phase speed when compared to sea level observations. Hence, considering the effect of the mean current on the estimation of the Rossby wave speed is likely to improve the model and extend the predictability.

\section{b. Predictability due to changes in isopycnal motion, spiciness, and processes in the mixed layer}

Temperature and salinity anomalies can be represented as active tracers (which can cause density changes;
Doney et al. 2007) and passive tracers (temperature and salinity density-compensating anomalies advected along streamlines of a geostrophic current; e.g., Munk 1981; Schneider 2000; Yeager and Large 2004). The anomalies that cause density changes are sometimes termed as heave anomalies (associated with displacement of isopycnals), while those that are compensated on a given isopycnal surface are termed as spice anomalies. Following this idea, a diagnostic method was proposed by Köhl (2014) to detect physical mechanisms leading to regional steric sea level change. We apply this decomposition approach by which the thermosteric and halosteric SSH changes are split into the spice ( $\mathrm{SSH}$ changes due to movement of spiciness along isopycnals) and isopycnal motion terms ( $\mathrm{SSH}$ changes due to displacement of isopycnals). The approach uses the assumption of small influence of mixing processes, therefore the spice and isopycnal motion terms are calculated from the depths 
below the mixed layer, whereas the contribution from the mixed layer is not further separated (mixed layer term):

$$
\begin{aligned}
\eta_{x}= & \eta_{x}^{\mathrm{MLD}}+\left(\eta_{x}^{\mathrm{IM}}+\eta_{x}^{\mathrm{spice}}\right) \\
= & -\int_{-\operatorname{maxMLD}}^{0} \alpha_{x} \Delta x d z \\
& -\int_{-H}^{-\operatorname{maxMLD}} \alpha_{x}\left(\frac{\nabla \bar{x} \cdot \nabla \bar{\gamma}}{\|\nabla \bar{\gamma}\|^{2}} \Delta \rho+\Delta x_{\rho}\right) d z,
\end{aligned}
$$

where $\eta_{x}^{\mathrm{MLD}}, \eta_{x}^{\mathrm{IM}}$, and $\eta_{x}^{\text {spice }}$ are the mixed layer, isopycnal motion, and spice terms, respectively, for the thermosteric SSH changes if $x$ is the temperature field or halosteric SSH changes if $x$ is the salinity field. The variable $\Delta \rho$ is the density anomaly, $\nabla \bar{\gamma}$ is the gradient of neutral density [defined according to McDougall (1987) to account for compressibility], and $\nabla \bar{x}$ is the gradient of the temperature (or salinity) field. The overbar denotes the time mean. The variable $\alpha_{x}= \pm\left(1 / \rho_{0}\right) \partial \rho / \partial x$ is the coefficient of expansion for temperature (salinity), and $\rho_{0}$ is a reference density. Depth levels $z=0$ and $z=-H$ represent the ocean surface and ocean floor, respectively. The maximum mixed layer depth (maxMLD; $0.125 \mathrm{~kg} \mathrm{~m}^{-3}$ criterion) is calculated as the largest monthly mean MLD values over the period 1952-2001. Because spice anomalies result from density-compensating temperature-salinity anomalies, the spice term can be calculated either from temperature or salinity changes.

The terms in the parentheses in Eq. (3) are the isopycnal motion term and the spice term. The temperature and salinity spiciness anomalies can be calculated as residuals to the projection on the mean density gradient: $\Delta x_{\rho}=\Delta x-\left[(\nabla \bar{x} \cdot \nabla \bar{\gamma}) /\|\nabla \bar{\gamma}\|^{2}\right] \Delta \rho$. Here, we calculate the spice term as follows: $\eta_{x}^{\text {spice }}=\eta_{x}-\eta_{x}^{\mathrm{MLD}}-\eta_{x}^{\mathrm{IM}}$

Köhl (2014) demonstrated that thermosteric SSH variability is largely related to isopycnal heaving, while halosteric variability mostly arises from spiciness. Thus, the expectation is that the skill of the thermosteric component relates to the isopycnal motion part. On the other hand, the additional mechanism of spiciness advection along isopycnals by the mean circulation may provide a mechanism by which the individual thermosteric and halosteric SSH changes exhibit larger skill than their sum, the steric SSH.

Since a large fraction of anomalies associated with isopycnal motion is believed to occur as adjustment to wind stress forcing through baroclinic Rossby waves, we expect that the isopycnal motion term will have similar patterns of skill as that of the RWM. The mixed layer term is expected to have at least the persistence skill due to heat content anomalies in the winter mixed layer (Köhl 2014). This is related to the mechanism introduced by Frankignoul and Hasselmann (1977) based on the persistence of ocean heat content in the mixed layer. Because of the advection of heat by mean circulation, the spice term might be predictable as well.

For yr2-5, Fig. 6 shows regions of significant predictive skill for the isopycnal motion and mixed layer terms for the thermosteric SSH and the skill for the spice term, which is relevant for both thermosteric and halosteric SSH changes. This figure shows the significant skill separated in regions with interannual variability (derived from the GECCO synthesis) smaller (larger) than $1.5 \mathrm{~cm}$ in orange (green). Regions of significant persistence skill are masked in red. The predictive skill in regions of large interannual variability exists for the thermosteric mixed layer term over the North Atlantic, for the isopycnal motion term over the subtropical Pacific Ocean and North Atlantic, and for the spice term over the subpolar North Atlantic.

In the Pacific Ocean, the skill for two contributions to steric $\mathrm{SSH}$ changes is present: the isopycnal motion term in the central North and South Pacific $\left(30^{\circ}-40^{\circ} \mathrm{N}\right.$ and $30^{\circ}-40^{\circ} \mathrm{S}$ ) and the spice term (persistence skill) in the eastern North Pacific and central South Pacific. Over the Indian Ocean, the persistence skill dominates the patterns for the heave term north of $10^{\circ} \mathrm{S}$, for the mixed layer term in the Arabian Sea, and for the spice term around $30^{\circ} \mathrm{S}$. In the North Atlantic, the skill from all three contributions is present. Persistence of heat content anomalies in the mode water regions plays only a small role. Since the mixed layer term encompasses also the regions that are only seldom mixed, a large fraction of the skill associated with the mixed layer term may actually be related to the other mechanism, particularly since the patterns of high skill are directly adjacent to those related to the other terms. Further predictive skill for the mixed layer term is evident in the Southern Ocean and the subtropical Atlantic up to yr6-9 (not shown). Over the subpolar North Atlantic, the spice term shows skill up to yr2-5.

Patterns of skill for the isopycnal motion term show some resemblance to the skill of the RWM in the North Pacific $\left(20^{\circ}-50^{\circ} \mathrm{N}\right)$ and the North Atlantic $\left(40^{\circ}-60^{\circ} \mathrm{N}\right)$. Differences are obvious in the Indian Ocean and the subtropical Atlantic, where the RWM does not provide skill because of the short transition time of the first baroclinic mode (about 1-2 years). However, although the first baroclinic mode is believed to be the most relevant response to wind stress, buoyancy forcing may force higher baroclinic modes (Thompson and Ladd 2004), which enable much slower propagation and may explain why even in low latitudes skill patterns become indistinguishable from the persistence. The pattern correlation coefficient, which suggests the level of correspondence between predictive skill for the isopycnal 

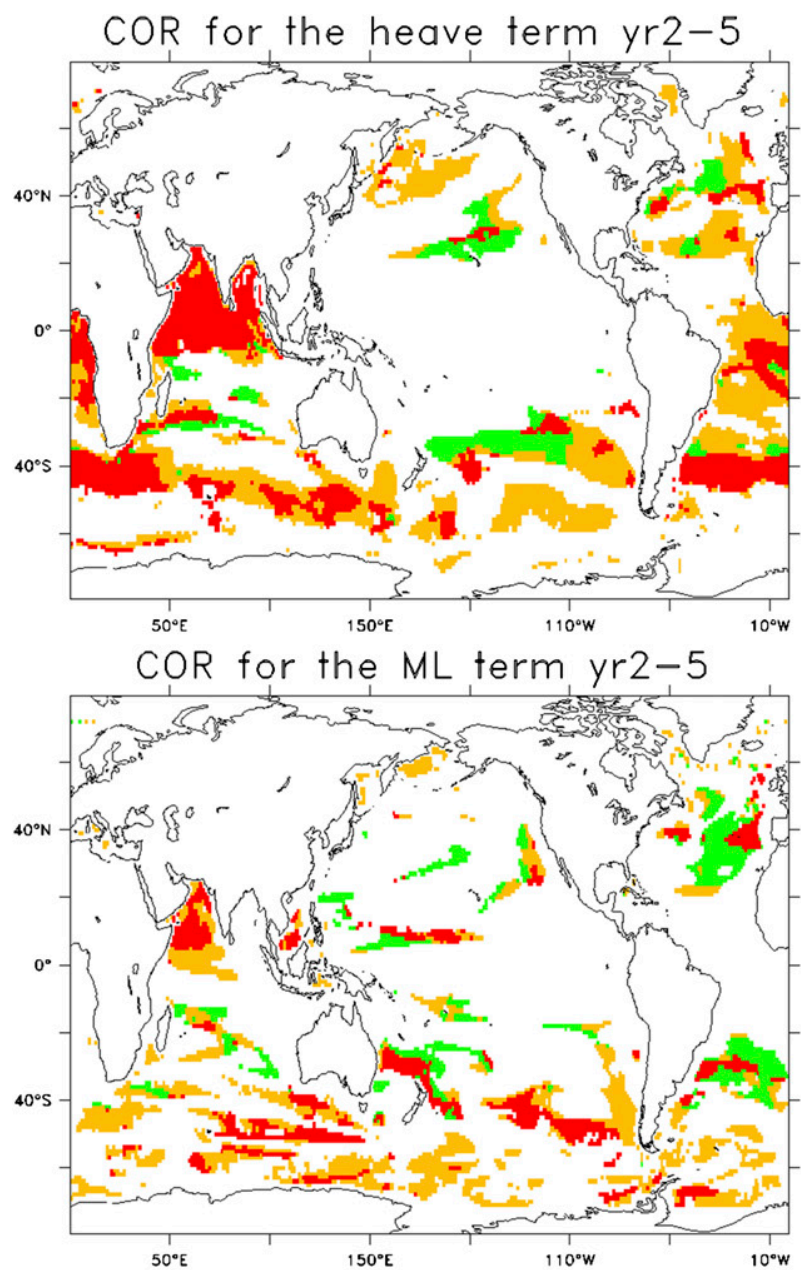

COR for the spice term yr2-5

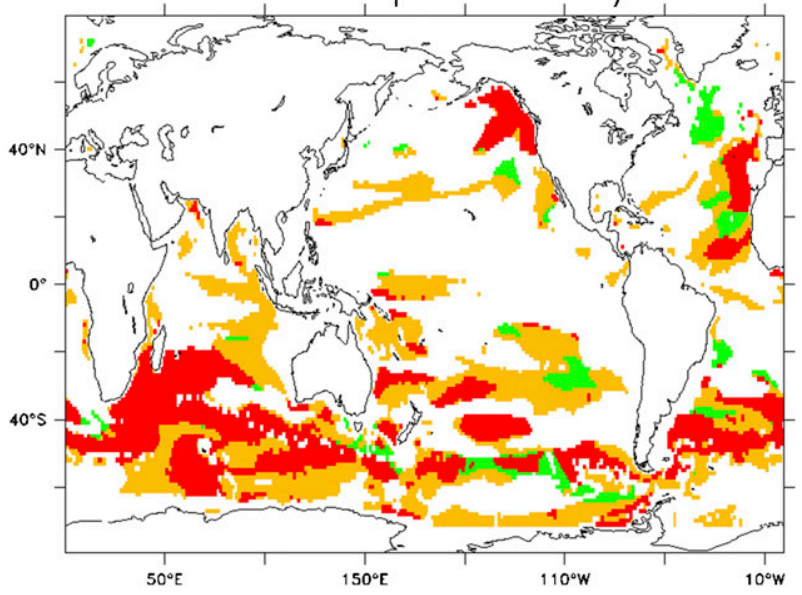

FIG. 6. Significant COR skill for three thermosteric SSH contributions: (top) isopycnal motion term, (middle) mixed layer term, and (bottom) spice term. COR is computed between the detrended signals from GECCO and the initialized hindcasts for yr2-5. Orange areas indicate significant COR; green areas show significant COR over the regions with the standard deviation values more than $1.5 \mathrm{~cm}$. Regions with significant persistence skill, which overlap with predictive skill, are masked in red. motion term and the skill from RWM, is about 0.18 north of $20^{\circ} \mathrm{N}\left(0.10\right.$ south of $\left.20^{\circ} \mathrm{S}\right)$. The correspondence is higher between the skill for steric SSH and its isopycnal motion contribution, about 0.28 north of $20^{\circ} \mathrm{N}$ $\left(0.35\right.$ south of $\left.20^{\circ} \mathrm{S}\right)$.

\section{Discussion and conclusions}

This study aims to identify areas where regional steric sea level changes show predictive skill and to determine underlying mechanisms up to decadal time scales. In particular, the study addresses the question of predictability for regional interannual steric SSH changes and its thermosteric and halosteric components. Though (because of short overlap time between SSH hindcasts and altimeter observations) the predictive skill throughout the study is mostly estimated with respect to the GECCO synthesis, the SSH hindcasts were also compared with respect to the tide gauge data. The analysis of skill based on GECCO neglects the errors in ocean initial conditions. The effect of errors on the predictive skill is seen when comparing the initialized hindcasts to the tide gauge dataset. The skill based on tide gauges is lower and vanishes faster than the skill based on GECCO. Initialized SSH hindcasts compared to the tide gauge data mostly showed skill in the first lead year, while for later lead years some skill is evident near $20^{\circ} \mathrm{S}$ and $20^{\circ} \mathrm{N}$ in the Pacific Ocean and in the Caribbean basin. Based on the GECCO synthesis, predictive skill for steric sea level is found over the subtropical Atlantic and Pacific Oceans, along the path of the North Atlantic Current, over the Indian and Southern Oceans.

The patterns of predictive skill for the steric signal are similar to those for dynamic topography (shown by Polkova et al. 2014) and suggest predictability for regional steric sea level changes up to decadal time scales. At a lead time longer than $1 \mathrm{yr}$, the patterns of predictive skill show more regions of significant correlation values than the persistence forecast. Both thermosteric and halosteric sea level changes demonstrate large-scale patterns of high predictive skill, whereas for thermosteric contributions, these skill patterns correspond to the regions of large interannual variability.

Since previous studies indicate that steric sea level changes are dominated by the redistribution of heat and freshwater in response to wind forcing, it might be expected that this wind-driven part of sea level, which manifests itself as isopycnal motion, is not predictable (Piecuch and Ponte 2011). However, Qiu and Chen (2006) showed that the wind-driven baroclinic Rossby wave model can explain the midlatitude SSH variability on interannual-to-decadal time scales. Anomalous wind stress curl leads via Ekman pumping to isopycnal motion and to sea level anomalies that propagate as baroclinic Rossby waves. Thus, if the 
steric sea level anomaly propagates as the baroclinic Rossby wave, some part of the steric sea level signal could be predictable. Köhl (2014) demonstrated that thermosteric SSH variability is dominated by isopycnal motion; thus, if this contribution is predictable, we could also predict thermosteric SSH changes.

Our results show that predictability of thermosteric SSH is associated with the isopycnal motion term over the subtropical Atlantic and Pacific, the Southern Ocean, and the Indian Ocean. Predictability of the isopycnal motion term in the subtropics at yr2-5 is related to the first baroclinic Rossby wave propagation. Mechanisms seem to be different in the tropics (e.g., Indian Ocean and the tropical Atlantic). For instance, Miles et al. (2014) demonstrated that seasonal predictions of sea level anomalies could be skillful in the tropics, and the skill is related to the Kelvin waves and by association with El Niño-Southern Oscillation and the Indian Ocean dipole. The heat content of the mixed layer is one of the dominant contributions to predictive skill for thermosteric SSH changes in the North Atlantic. Predictive skill for the mixed layer over the North Atlantic might be related to the advection of heat anomalies poleward by the Atlantic meridional overturning circulation (e.g., Latif and Keenlyside 2011; Msadek et al. 2010), which in the current setup demonstrates predictive skill up to yr6-9 (Polkova et al. 2014).

In contrast, the halosteric SSH variability is largely dominated by the advection of spiciness, which was expected to be predictable as it is related to the mean circulation (Köhl 2014). The halosteric SSH shows large areas of skill that are mostly the regions of low interannual variability (about $2 \mathrm{~cm}$ ), with the exception of the subpolar North Atlantic (about $10 \mathrm{~cm}$ ). The advection of spiciness, which is expected to provide predictability for the halosteric SSH, showed large areas of skill in the subpolar and eastern North Atlantic, subtropical South Pacific, and the extratropical Southern Hemisphere. In the subtropical gyre of the North Atlantic, the advection of temperature and salinity anomalies was studied earlier by Laurian et al. (2009), who argued that the strongest spiciness anomalies develop in the western part of the salinity maximum region. The spiciness is often generated from the mixed layer by subduction of temperature and salinity properties along the isopycnals (Yeager and Large 2004). Hence, anomalies in spiciness and within the mixed layer can be affected by the same mechanism, but the spiciness anomalies could propagate further independently. However, in the current study, the predictability related to spiciness showed large areas of persistence skill, probably due to slow advection speeds.

The low number of ensemble members and the low number of starting dates (in our case, three ensemble members and initialization every five years, the minimum numbers suggested for CMIP5 experimental setup; Taylor et al. 2012) lead to lower skill and higher significance thresholds than what is available from some other hindcast sets. However, the robustness of the analysis was tested on the GECCO2 initialized hindcasts that recently became available from the Mittelfristige Klimaprognosen (MiKlip) project (http://www.fona-miklip. de). The hindcasts have larger ensemble size ( 15 members) and are initialized every year. The results showed comparable patterns of skill considering every 5- and 1-yr initialization, with the latter having lower predictive skill in the tropics. However, because of the larger verification sample, and hence lower significance threshold, the predictive skill in the tropics becomes significant.

Acknowledgments. This work was supported in part by the Max Planck Fellow Program through the project "Coupled Climate System Data Assimilation" and through the BMBF-funded projects RACE and MiKlip. All model simulations were performed at the German Climate Computing Centre (DKRZ). Contribution to the DFG funded excellence cluster CliSAP of the Universität Hamburg (Research Area A03). We also thank to three anonymous reviewers whose comments helped us to improve the manuscript.

\section{REFERENCES}

Agarwal, N., A. Köhl, C. R. Mechoso, and D. Stammer, 2014: On the early response of the climate system to a meltwater input from Greenland. J. Climate, 27, 8276-8296, doi:10.1175/ JCLI-D-13-00762.1.

Boer, G., V. Kharin, and W. Merryfield, 2013: Decadal predictability and forecast skill. Climate Dyn., 41, 1817-1833, doi:10.1007/s00382-013-1705-0.

Cabanes, C., T. Huck, and A. Colin de Verdière, 2006: Contributions of wind forcing and surface heating to interannual sea level variations in the Atlantic Ocean. J. Phys. Oceanogr., 36, 1739-1750, doi:10.1175/JPO2935.1.

Capotondi, A., and M. Alexander, 2001: Rossby waves in the tropical North Pacific and their role in decadal thermocline variability. J. Phys. Oceanogr., 31, 3496-3515, doi:10.1175/ 1520-0485(2002)031<3496:RWITTN > 2.0.CO;2.

Cazes-Boezio, G., D. Menemenlis, and C. Mechoso, 2008: Impact of ECCO ocean-state estimates on the initialization of seasonal climate forecasts. J. Climate, 21, 1929-1947, doi:10.1175/ 2007JCLI1574.1.

Chelton, D. B., R. A. Deszoeke, M. G. Schlax, K. El Naggar, and N. Siwertz, 1998: Geographical variability of the first baroclinic Rossby radius of deformation. J. Phys. Oceanogr., 28, 433-460, doi:10.1175/1520-0485(1998)028<0433:GVOTFB >2.0.CO;2.

Church, J. A., P. L. Woodworth, T. Aarup, and W. S. Wilson, Eds., 2010: Understanding Sea-Level Rise and Variability. Wiley, 428 pp.

Delworth, T. L., and T. R. Knutson, 2000: Simulation of early 20th century global warming. Science, 287, 2246-2250, doi:10.1126/ science.287.5461.2246.

Doney, S. C., S. Yeager, G. Danabasoglu, W. G. Large, and J. C. McWilliams, 2007: Mechanisms governing interannual variability 
of upper-ocean temperature in a global ocean hindcast simulation. J. Phys. Oceanogr., 37, 1918-1938, doi:10.1175/ JPO3089.1.

Frankignoul, C., and K. Hasselmann, 1977: Stochastic climate models, Part II Application to sea-surface temperature anomalies and thermocline variability. Tellus, 29, 289-305, doi:10.1111/ j.2153-3490.1977.tb00740.x.

Fu, L.-L., and B. Qiu, 2002: Low-frequency variability of the North Pacific Ocean: The roles of boundary- and wind-driven baroclinic Rossby waves. J. Geophys. Res., 107, 3220, doi:10.1029/ 2001JC001131.

Goddard, L., and Coauthors, 2013: A verification framework for interannual-to-decadal predictions experiments. Climate Dyn., 40, 245-272, doi:10.1007/s00382-012-1481-2.

Griffies, S., and K. Bryan, 1997: A predictability study of simulated North Atlantic multidecadal variability. Climate Dyn., 13, 459-487, doi:10.1007/s003820050177.

Hawkins, E., J. Robson, R. Sutton, D. Smith, and N. Keenlyside, 2011: Evaluating the potential for statistical decadal predictions of sea surface temperatures with a perfect model approach. Climate Dyn., 37, 2495-2509, doi:10.1007/ s00382-011-1023-3.

Huber, M., and R. Knutti, 2011: Anthropogenic and natural warming inferred from changes in Earth's energy balance. Nat. Geosci., 5, 31-36, doi:10.1038/ngeo1327.

Ivchenko, V. O, S. Danilov, D. Sidorenko, J. Schröter, M. Wenzel, and D. L. Aleynik, 2008: Steric height variability in the Northern Atlantic on seasonal and interannual scales. J. Geophys. Res., 113, C11007, doi:10.1029/2008JC004836.

Kalnay, E., and Coauthors, 1996: The NCEP/NCAR 40-Year Reanalysis Project. Bull. Amer. Meteor. Soc., 77, 437-471, doi:10.1175/ 1520-0477(1996)077<0437:TNYRP $>2.0$. CO;2.

Killworth, P. D., and J. R. Blundell, 2003a: Long extratropical planetary wave propagation in the presence of slowly varying mean flow and bottom topography. Part I: The local problem. J. Phys. Oceanogr., 33, 784-801, doi:10.1175/1520-0485(2003)33<784: LEPWPI $>2.0 . \mathrm{CO} ; 2$.

$\longrightarrow$, and,$- 2003 \mathrm{~b}$ : Long extratropical planetary wave propagation in the presence of slowly varying mean flow and bottom topography. Part II: Ray propagation and comparison with observations. J. Phys. Oceanogr., 33, 802-821, doi:10.1175/ 1520-0485(2003)33<802:LEPWPI > 2.0.CO;2.

Kim, H.-M., P. J. Webster, and J. A. Curry, 2012: Evaluation of short-term climate change prediction in multi-model CMIP5 decadal hindcasts. Geophys. Res. Lett., 39, L10701, doi:10.1029/ 2012GL051644.

Köhl, A., 2014: Detecting the origin of interannual steric sea level changes. J. Climate, 27, 2417-2426, doi:10.1175/ JCLI-D-13-00412.1.

- , and D. Stammer, 2008a: Decadal sea level changes in the 50-year GECCO ocean synthesis. J. Climate, 21, 1876-1890, doi:10.1175/2007JCLI2081.1.

__ and _ - 2008b: Variability of the meridional overturning in the North Atlantic from the 50-year GECCO state estimation. J. Phys. Oceanogr., 38, 1913-1930, doi:10.1175/ 2008JPO3775.1.

Kröger, J., W. A. Müller, and J.-S. von Storch, 2012: Impact of different ocean reanalyses on decadal climate prediction. Climate Dyn., 39, 795-810, doi:10.1007/s00382-012-1310-7.

Latif, M., and T. P. Barnett, 1996: Decadal climate variability over the North Pacific and North America: Dynamics and predictability. J. Climate, 9, 2407-2423, doi:10.1175/1520-0442(1996)009<2407: DCVOTN $>2.0 . \mathrm{CO} ; 2$
_ variability and predictability. Deep-Sea Res. II, 58, 1880-1894, doi:10.1016/j.dsr2.2010.10.066.

Laurian, A., A. Lazar, and G. Reverdin, 2009: Generation mechanism of spiciness anomalies: An OGCM analysis in the North Atlantic subtropical gyre. J. Phys. Oceanogr., 39, 1003-1018, doi:10.1175/2008JPO3896.1.

Ma, H.-Y., C. R. Mechoso, Y. Xue, H. Xiao, J. D. Neelin, and X. Ji, 2013: On the connection between continental-scale land surface processes and the tropical climate in a coupled oceanatmosphere-land system. J. Climate, 26, 9006-9025, doi:10.1175/ JCLI-D-12-00819.1.

Magnusson, L., M. Alonso-Balmaseda, S. Corti, F. Molteni, and T. Stockdale, 2013: Evaluation of forecast strategies for seasonal and decadal forecasts in presence of systematic model errors. Climate Dyn., 41, 2393-2409, doi:10.1007/ s00382-012-1599-2.

Marshall, J., A. Adcroft, C. Hill, L. Perelman, and C. Heisey, 1997: A finite-volume, incompressible Navier Stokes model for studies of the ocean on parallel computers. J. Geophys. Res., 102, 5753-5766, doi:10.1029/96JC02775.

Matei, D., H. Pohlmann, J. Jungclaus, W. Müller, H. Haak, and J. Marotzke, 2012: Two tales of initializing decadal climate prediction experiments with the ECHAM5/MPI-OM model J. Climate, 25, 8502-8523, doi:10.1175/JCLI-D-11-00633.1.

McDougall, T. J., 1987: Neutral surfaces. J. Phys. Oceanogr., 17, 19501964, doi:10.1175/1520-0485(1987)017<1950:NS > 2.0.CO;2.

Mechoso, C. R., J.-Y. Yu, and A. Arakawa, 2001: A coupled GCM pilgrimage: From climate catastrophe to ENSO simulations. Circulation Model Development, D. A. Randall, Ed., International Geophysics Series, Vol. 70, Elsevier, 539-575, doi:10.1016/ S0074-6142(00)80066-2.

Miles, E. R., C. M. Spillman, J. A. Church, and P. C. McIntosh, 2014: Seasonal prediction of global sea level anomalies using an ocean-atmosphere dynamical model. Climate Dyn., 43, 2131-2145, doi:10.1007/s00382-013-2039-7.

Msadek, R., K. Dixon, T. Delworth, and W. Hurlin, 2010: Assessing the predictability of the Atlantic meridional overturning circulation and associated fingerprints. Geophys. Res. Lett., 37, L19608, doi:10.1029/2010GL044517.

Munk, W., 1981: Internal waves and small-scale processes. Evolution of Physical Oceanography, B. A. Warren and C. Wunsch, Eds., MIT Press, 264-291.

Pattullo, J., W. Munk, R. Revelle, and E. Strong, 1955: The seasonal oscillation in sea level. J. Mar. Res., 14, 88-155.

Piecuch, C., and R. Ponte, 2011: Mechanisms of interannual steric sea level variability. Geophys. Res. Lett., 38, L15605, doi:10.1029/ 2011GL048440.

Pierce, D., T. Barnett, R. Tokmakian, A. Semtner, M. Maltrud, J. Lysne, and A. Craig, 2004: The ACPI project, element 1: Initializing a coupled climate model from observed conditions. Climatic Change, 62, 13-28, doi:10.1023/B:CLIM.0000013676.42672.23.

Pohlmann, H., J. Jungclaus, A. Köhl, D. Stammer, and J. Marotzke, 2009: Initializing decadal climate predictions with the GECCO oceanic synthesis: Effects on the North Atlantic. J. Climate, 22, 3926-3938, doi:10.1175/2009JCLI2535.1.

_ D. M. Smith, M. A. Balmaseda, N. S. Keenlyside, S. Masina, D. Matei, W. A. Müller, and P. Rogel, 2013: Predictability of the mid-latitude Atlantic meridional overturning circulation in a multi-model system. Climate Dyn., 41, 775-785, doi:10.1007/ s00382-013-1663-6.

Polkova, I., A. Köhl, and D. Stammer, 2014: Impact of initialization procedures on the predictive skill of a coupled ocean- 
atmosphere model. Climate Dyn., 42, 3151-3169, doi:10.1007/ s00382-013-1969-4.

Ponte, R. M., 2006: Low-frequency sea level variability and the inverted barometer effect. J. Atmos. Oceanic Technol., 23, 619629, doi:10.1175/JTECH1864.1.

Qiu, B., and S. Chen, 2006: Decadal variability in the large-scale sea surface height field of the South Pacific Ocean: Observations and causes. J. Phys. Oceanogr., 36, 1751-1762, doi:10.1175/ JPO2943.1.

Santer, B., and Coauthors, 2011: Separating signal and noise in atmospheric temperature changes: The importance of timescale. J. Geophys. Res., 116, D22105, doi:10.1029/2011JD016263.

Schneider, N., 2000: A decadal spiciness mode in the tropics. Geophys. Res. Lett., 27, 257-260, doi:10.1029/1999GL002348.

Smith, D. M., S. Cusack, A. Colman, C. Folland, G. Harris, and J. Murphy, 2007: Improved surface temperature prediction for the coming decade from a global climate model. Science, $\mathbf{3 1 7}$ 796-799, doi:10.1126/science.1139540.

-, R. Eade, and H. Pohlmann, 2013: A comparison of full-field and anomaly initialization for seasonal to decadal climate prediction. Climate Dyn., 41, 3325-3338, doi:10.1007/s00382-013-1683-2.

Stammer, D., N. Agarwal, P. Herrmann, A. Köhl, and C. Mechoso, 2011: Response of a coupled ocean-atmosphere model to Greenland ice melting. Surv. Geophys., 32, 621-642, doi:10.1007/ s10712-011-9142-2.

—, A. Cazenave, R. M. Ponte, and M. E. Tamisiea, 2013: Causes for contemporary regional sea level changes. Annu. Rev. Mar. Sci., 5, 21-46, doi:10.1146/annurev-marine-121211-172406.

Stockdale, T., 1997: Coupled ocean-atmosphere forecasts in the presence of climate drift. Mon. Wea. Rev., 125, 809-818, doi:10.1175/1520-0493(1997)125<0809:COAFIT>2.0.CO;2.
Sturges, W., B. Hong, and A. J. Clarke, 1998: Decadal wind forcing of the North Atlantic subtropical gyre. J. Phys. Oceanogr., 28, 659668, doi:10.1175/1520-0485(1998)028<0659:DWFOTN >2.0.CO;2.

Taylor, K. E., R. J. Stouffer, and G. A. Meehl, 2012: An overview of CMIP5 and the experiment design. Bull. Amer. Meteor. Soc., 93, 485-498, doi:10.1175/BAMS-D-11-00094.1.

Thompson, L. A., and C. A. Ladd, 2004: The response of the North Pacific Ocean to decadal variability in atmospheric forcing: Wind versus buoyancy forcing. J. Phys. Oceanogr., 34, 1373-1386, doi:10.1175/1520-0485(2004)034<1373:TROTNP>2.0.CO;2.

Vinogradova, N., R. Ponte, and D. Stammer, 2007: Relation between sea level and bottom pressure and the vertical dependence of oceanic variability. Geophys. Res. Lett., 34, L03608, doi:10.1029/2006GL028588.

Weijer, W., E. Muñoz, N. Schneider, and F. Primeau, 2013: Pacific decadal variability: Paced by Rossby basin modes? J. Climate, 26, 1445-1456, doi:10.1175/JCLI-D-12-00316.1.

Yang, H., A. Hugh, and Z. Liu, 2003: Basin modes in a tropicalextratropical basin. J. Phys. Oceanogr., 33, 2751-2763, doi:10.1175/ 1520-0485(2003)033<2751:BMIATB $>2.0 . C O ; 2$

Yeager, S. G., and W. G. Large, 2004: Late-winter generation of spiciness on subducted isopycnals. J. Phys. Oceanogr., 34, 1528-1547, doi:10.1175/1520-0485(2004)034<1528: LGOSOS $>2.0 . \mathrm{CO} ; 2$.

—, A. Karspeck, G. Danabasoglu, J. Tribbia, and H. Teng, 2012: A decadal prediction case study: Late twentieth-century North Atlantic Ocean heat content. J. Climate, 25, 5173-5189, doi:10.1175/ JCLI-D-11-00595.1.

Zhang, X., and J. A. Church, 2012: Sea level trends, interannual and decadal variability in the Pacific Ocean. Geophys. Res. Lett., 39, L21701, doi:10.1029/2012GL053240. 